\title{
THE ROLE OF SYNAPSIN I IN INTRACELLULAR SIGNALING MECHANISMS UNDERLIES EXERCISE-INDUCED IMPROVEMENT IN SOCIAL MEMORY: A SYSTEMATIC REVIEW
}

\author{
RIA MARGIANA ${ }^{1}$, AKMAL PRIMADIAN SUPRAPTO2* \\ ${ }^{1}$ Department of Anatomy, Faculty of Medicine, University of Indonesia, Jakarta, Indonesia. ${ }^{2}$ Medical Doctor, Kemayoran Public Hospital, \\ Jakarta, Indonesia. Email: akmal.primadian@alumni.ui.ac.id \\ Received: 12 April 2017, Revised and Accepted: 30 May 2017
}

\section{ABSTRACT}

Objective: Intracellular signaling mechanism is an important biological function, as scholars continue to seek new ways of improving social memory. Researchers have conducted several studies on the role of synapsin I in intracellular signaling mechanism. This study assessed the empirical evidence that shows the role of synapsin I in intracellular signaling mechanism with the aim of achieving exercise-induced improvement in social memory.

Methods: Nine previously conducted researches were reviewed in this paper. The included studies were controlled laboratory experiments involving mice as the subjects.

Results: Although the studies included were done in different timelines, the researchers agreed in unison that synapsin I plays a crucial role in cell signaling. The outcome of the practical studies was vital in understanding function and physiology of human cells, which is fundamental in science and human anatomy.

Conclusion: In particular, the findings shows how exercise can improve social memory by triggering the intracellular signaling mechanism. The limited number of studies addressing the topic of intracellular cell signaling suggests that more study is needed to provide more evidence on the issue.

Keywords: Synapsin I, Intracellular signaling, Exercise, Social memory.

(C) 2017 The Authors. Published by Innovare Academic Sciences Pvt Ltd. This is an open access article under the CC BY license (http://creativecommons. org/licenses/by/4. 0/) DOI: http://dx.doi.org/10.22159/ajpcr.2017.v10i9.19111

\section{INTRODUCTION}

Physical exercise, is by far, one of the most powerful and efficient cerebral motivators. Apparently, exercise is capable of inducing significant and rather reliable developments in performance in various mental activities among variable species. Most mental tasks take place in the brain with the neurotropic and neurogenic those are facilitated by exercise. Critical studies relate to results of brain-derived neurotrophic factor (BDNF) as the sole mediator of the positive impacts of exercise concerning the aspects of cognition and neurogenesis [1,2]. An underlying role for the BDNF in these exercise controlled transformations has not necessarily been established. More so, the individual techniques through which the supposed activities augment the expression of BDNF in the brain and by which these apparent increases transform into memory developments remain unknown [3]. As researchers continue to discover and develop new medicines, it is necessary to shed more light on intracellular cell signaling. Understanding how synapsin I affects the signaling mechanism can assist in developing new pathways for addressing medical issues that affect the body's functionality at the cellular level. Critical examination and synapsis effect with exercise improves the social memory implying relevant studies. The findings and conclusion are the same in this study.

Evidently, the part played by the BDNF in learning and memory has been expressly determined as regards the individual short-term and long-lasting impacts on the neurons [1]. The effects may well undergo alleviation by its apparent obligatory connection to the tyrosine kinase $B$ receptor and the subsequent initiation of flexibility-affiliated signaling flow [4]. One of the signaling flows is the extracellular signal-affiliated kinase channel known to be imperative in some methods of learning and memory. Key studies encompassed the particular examination of the effects of induced exercise on the manifestation and discharge of BDNF and the initiation of the BDNF receptor in the dentate gyrus [4]. Nonetheless, they entailed the investigation of set manipulation tactics about the control for impacts of learning on cell motioning and or signaling. Nevertheless, their revelations ascertain that exercise has the capability to incite additional transformation in the hippocampus that may well trigger the development of social memory through such aspects as regards the rise in the manifestation of vesicle proteins of the synapse-like synapsin I, as well as in the neurogenesis [5].

\section{METHODS}

This paper recruited studies done in the past 10 years on the role of synapsin I in intracellular signaling mechanism. The idea was to identify studies done in a laboratory setting that provide evidence that can address the objective of this study. The researcher identified several eligibility criteria for the inclusion of articles in the study. The steps to review were explained in the Fig. 1. First, the study had to be peerreviewed. The PROQUEST online database provided for the filtration of peer-reviewed articles only. The study relied majorly on laboratory experiments, and the researchers majored their projects on rats as the specimen. The idea was to seek uniformity of the study that allowed comparison of findings. The literature searching was conducted by surfing into the PROQUEST database to identify articles relevant for the study. The online database provides for the identification of other studies conducted by the same authors. After logging into the PROQUEST website, the researcher set the setting to "peer-reviewed." The years ranged from 2005 to 2015, which separated the old study from research references. Finally, the researcher entered the keywords "Synapsin I" and "Intracellular Signaling Mechanisms" and pressed the search button.

The idea was to use the summary that the abstracts provided to determine the relevance of the studies. The review of abstract allowed the research to open and review the entire study after it passed the criteria. After the relevant studies were identified, the relevant data were extracted. The reviewed study shows the information pertaining 


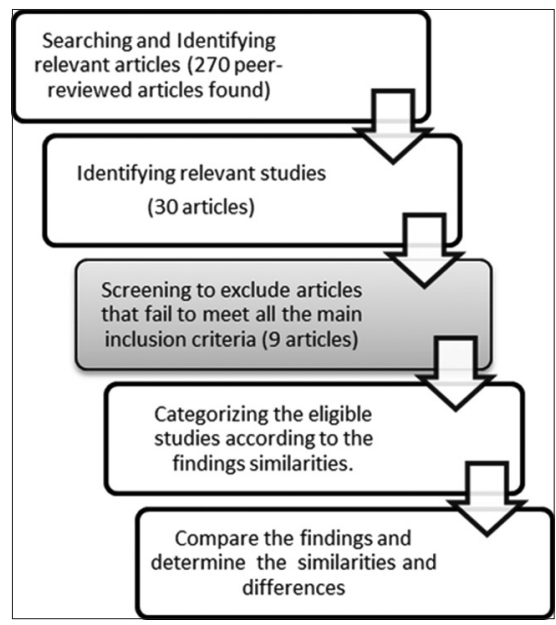

Fig. 1: The systematic selection of studies

the authors detail included with a year of publication and the mode of study. The mode of study and the results is organized in Table 1. Articles were carefully selected that involved this paper making in addressing the predetermined focus. The abstract was reviewed after the study was collected.

In most of the studies that were mentioned in Table 1 , mice were exterminated by beheading almost with immediate effect after completing a certain set of activities appertaining to specific behavioral dispositions plus the individual brains were dispensed with and dissected hemispherical-wise on ice [6]. In this regard, the left half was assessed for immunohistochemistry. The resultant half was then situated on a respective cork disk covered with relevant compounds and afterward dissolved in liquid nitrogen. The frozen samples were in turn wrapped in aluminum foil and then stored in a temperature enhanced environment at $-80^{\circ} \mathrm{C}$ until such time as was appropriate for precise sectioning. Moreover, the right half section was subdivided through such techniques as the application of handicraft on ice and the particular dentate gyrus was then separated into three parts of equal measure for extensive consideration and or evaluation of the test subjects [7]. One of the portions of the sample was in turn cross cut for examination of the subsequent discharge of the BDNF. The secondary phase was in effect subjected effective storage measures as the preservation of the test subjects in ribonucleic acid later on in training for the purposes of RNA abstraction. Consistently, the third portion was forthrightly standardized in a buffer characteristically of lysis. Afterward, a cluster of the pure breed caged and controlled mice was evaluated in contrast for such purposes appertaining to the discharge of the respective BDNF discharge mechanisms [8]. The mice were assessed in terms of establishing and/or determining the notion of selectively evaluation of the characteristic traits of the two clusters of the test subjects, say the sedentary and active rats. The research entailed the inclusion of extra mice in storage seeing as though in the off-chance that a test subject should die in the process of investigation then extra mice could probably be used as an effective replacement [9]. The ethical considerations were assimilated in the conduction of the study.

The researcher conducts the study but avoided the aspect of animal test moral. Mice being the specimen in this case. The research of establishing the roles of synapsin 1 on the test mice may well entail the application of such moral laws that dictate the handling of the animals used as test subjects used in this study. Quite so, the analysts who did more in the way of coming up with somewhat conclusive findings regarding the investigation operated under the stringent confines of enacting the specific and or distinct moral concepts [10]. Nonetheless, the data sources utilized in the study were well explored as they were legitimate in ensuring that the medical aspects were, for all purposes and intent,
Table 1: The systematic review of the study

\begin{tabular}{lll}
\hline Author, year & Subjects & The research design \\
\hline Awizio et al. [13] 2007 & Mice & Laboratory experiment \\
Marais et al. [12] 2009 & Mice & Laboratory experiment \\
Fornasiero et al. [8] 2010 & Rats & Experimental research \\
Micheva et al.[14] 2010 & Rats & Laboratory experiment \\
Soontornniyomkij et al. [15] 2010 & Mice & Experimental research \\
Sharma et al. [16] 2010 & Wistar & Experimental research \\
& rats & \\
Li et al. [17] 2013 & Rats & Laboratory experiment \\
Fernandes et al. [3] 2013 & Mice & Laboratory experiment \\
Ohia-Nwoko et al. [7] 2014 & Mice & Laboratory experiment \\
\hline
\end{tabular}

corresponding with the rather stated and standard declarations put forth concerning the safety in handling animals seeing as though the animals [11]. In this regard, are not susceptible to deploying such physical endowment capabilities like the necessary awareness attributed to conscientious factors.

\section{RESULTS}

As indicated earlier, one of the main inclusion criteria for this study was the laboratory experiments involving rats. Therefore, the study incorporated such data collective tactics that involved the scrutinized examination of the test subjects - in this regard, the mice. In the selected study, the researchers had conducted a similar assessment of the rats' traits that relate to the intracellular signaling mechanism with regard to the synapsin I. The choice provided a common platform for comparison of the intracellular signaling mechanism. In all the 12 studies, the scholars agreed in unison that synapsin I plays a significant role in initiating the intracellular signaling mechanism. In one study, 1 week long of enforced physical exercise has the capacity of improving the social memory in a characteristically troubling dimensional activity appertaining to the augmentation of the corresponding to the manifestation of the brain derivative neurological factor in the dentate gyrus, hippocampus, and the perirhinal cortex [12]. The other studies revealed that the administration of a single dosage of BDNF in the intracellular cerebral ventricle is adequate to emulate the developments in the object identification. The findings revealed that apparently the test subjects mainly the exercised and sedentary species consistently displayed contrasting conceptual and or conclusive findings regarding the role of synapsin I in exercise-induced developments of individual mice.

The aspects appertaining the tested mice training and effective motivates approach creates response and activation. The findings also incorporated a final evaluation of the sedentary mice that equally matched the observatory experiences of the study. Suffice it to say; the study came up with such presuppositions as entail such findings appertaining to the influence of inactivity regarding establishing and or determining the role of synapsin I in the prospects of induced exercise toward affecting the social and or spatial memory.

Other researches covered the analysis of cell division and the indication of synapsin I in the dentate gyrus of the supposed mice. To find relevant and conclusive results, they covered methodologies pertaining rigorous and incorporation in the process of exercising social memory. In a research carried out by researchers using 3-month-old mice of $250 \mathrm{~g}$ from a highly reputable higher learning institute. The mice were grouped in three and provided with the basic of food and water. The rats experienced an equal amount of regulated timeline of light and dark sequences. The mice were placed in an environment, which sustained controlled temperatures of between $20^{\circ} \mathrm{C}$ and $22^{\circ} \mathrm{C}$. The experiments were carried out under the confines of the stringent national law, as it was [3]. The exercise routine entailed the incorporation of methods, which dealt with the familiarization of the rats to motorized treadmills. The mice were in turn subjected to run on the supposed treadmill for a maximum of 15 minutes on two consecutive days [18]. The belt and conveyor speeds were regulated to 5-10 m per minute. 
The mice were then allocated to two distinct clusters mainly; an exercising group and a respective inactive group, each with six rats. Manipulation of timeline and operation was selected as an enforced exercise was also involved. The regimen constituted of a maximum of 7 days of treadmill running for $1 \mathrm{hr}$ per day at belt velocities of between 10 and $15 \mathrm{~m}$ per minute [19]. The used treadmill is fortified with wire loops in one end of the belt through which a weak electric shock can be provided. The shock served to influence the mice to run constantly and was initiated at lower rates of the average intensity of two on a particular scale of 10 , which corresponds to a current of 0.7 mill amperes with a specified pulse period of 2 seconds throughout all training days. The mice were monitored while training to make certain that they ran consistently and to check for evidence of any stress signals. The mice on sedentary groups were engaged in stationary treadmills with shock wire loops initiated at low rates for similar timelines [19]. The important exercise in object displacement activity was done on the last day of the training regimen and or routine [20].

According to the study, the mice sustained intraperitoneal administrations of $50 \mathrm{mg}$ per $\mathrm{kg}$ of Bride during the 1-week period of enforced training [21]. The chemical was incorporated in a precise culture containing a significant concentration of sodium chloride. The solution was subject to stringent sterilization and/or disinfection right before the administration of the injection. 50 minutes before being placed on the treadmills, the animals sustained the drugs.

\section{DISCUSSION}

The results of the research and access displayed from the supposed week old evaluation of enforced training can attribute some set developmental aspects as regards the development of social, mental aspects. The assessment of the study entailed the usage of object displacement activity in the examination of young and healthy mature mice in congruence with earlier and conclusive findings in the relevant laboratory [5]. Other findings in literary aspects presuppose that for short lasting training to improve social memory development; a dearth must be presently displayed. The activity of displacing the object expressly authenticates the load appertaining to the specific load that is variegated by sudden alteration of the training timelines. The study has presupposed that the inactive mice possess the capability of getting to learn less troubling tasks concerning the variation of this apparent activity. For example, the induction of specified training sessions of three by 5 minutes and two by 5 minutes while they are incapable of learning the findings of say, one by 5 minutes-timeline incorporated in the present research. Therefore, any sound medical venture and or analytical perspective in the societal outlook resulting from the supposed particular argument; it is rather to presuppose that the medical ascertains the relevant pros and cons of the medical affairs of individual test subjects. As outlined in the prospects of conducting a research, the set economic factors have to be considered rather seriously owing to the notion the economic status quo is in line with the examination of respective and or relative test subjects with an aim to disclose and or dispel with the set obstacle that may well occur as a deterrent. Precisely, the research explores the health wise declaration of the active roles of the synapsin I in the evaluation of the rats concerning the influence of induced training toward the familiarization of the test subjects to the foreign environments.

\section{Limitations}

This paper had several limitations that strained the ability of the researcher to present statistical evidence for each of the selected study. Evidently, few researchers have conducted controlled experiments about the topic. Therefore, the researcher had to work with few study since most of the studies on synapsin I were not touching on intracellular signaling mechanism. It is also worth noting that the study involved a sensitive and complicated research involving part of the human brain. To make conclusion and summary about the human brain, the researchers greatly relied on the mice to gain the project results.

\section{CONCLUSION}

In summary, there is solid evidence on the intracellular signaling mechanism, which underlies exercise-induced improvement in social memory. The choice of previously conducted laboratory experiments offered an excellent opportunity for comparison. As noted above, the experiments on rats revealed the critical role that synapsin I plays in intercellular signaling mechanism. Exercised and BDNF- treated rats exhibited a characteristic display of successfully completing individual tasks of object displacement - and the remarkable result of spatial learning. Mice subjected to exercise, and BDNF treatment showed increased incidences of BDNF manifestation while exercised rats registered similar rates of increased cellular division, catalyzed BDNF discharge as well as a synapsin I manifestation in the dentate gyrus. Suffice it to say; applied induced exercise augments of BDNF in the dentate gyrus are capable of installing developments in social memory through such aspects as regards the activation of signaling mechanisms that influence the transmission of synapses in the hippocampus.

\section{ACKNOWLEDGMENT}

Special thanks to Dr. Fera Ibrahim and Dr. Budiman Bella that has allowed the author to do the research in IHVCB Universitas Indonesia.

\section{REFERENCES}

1. Kim H, Heo HI, Kim DH, Ko IG, Lee SS, Kim SE, et al. Treadmill exercise and methylphenidate ameliorate symptoms of attention deficit/hyperactivity disorder through enhancing dopamine synthesis and brain-derived neurotrophic factor expression in spontaneous hypertensive rats. Neurosci Lett 2011;504(1):35-9.

2. Fogelman D, Zafonte R. Exercise to enhance neurocognitive function after traumatic brain injury. PM R 2012;4(11):908-13.

3. Fernandes J, Baliego LG, Peixinho-Pena LF, de Almeida AA, Venancio DP, Scorza FA, et al. Aerobic exercise attenuates inhibitory avoidance memory deficit induced by paradoxical sleep deprivation in rats. Brain Res 2013;1529:66-73.

4. Triviño-Paredes J, Patten AR, Gil-Mohapel J, Christie BR. The effects of hormones and physical exercise on hippocampal structural plasticity. Front Neuroendocrinol 2016;41:23-43.

5. Janke K. Voluntary Wheel Running Alters Brain-Derived Neurotrophic Factor Levels in the Hippocampus of Senescence Accelerated Mice (Doctoral Dissertation, Bowling Green State University).

6. Venezia AC, Guth LM, Spangenburg EE, Roth SM. Lifelong parental voluntary wheel running increases offspring hippocampal Pgc-1a mRNA expression but not mitochondrial content or Bdnf expression. Neuroreport 2015;26(8):467-72.

7. Ohia-Nwoko O, Montazari S, Lau YS, Eriksen JL. Long-term treadmill exercise attenuates tau pathology in P301S tau transgenic mice. Mol Neurodegener 2014;9(1):54

8. Fornasiero EF, Bonanomi D, Benfenati F, Valtorta F. The role of synapsins in neuronal development. Cell Mol Life Sci 2010;67(9):1383-96.

9. Holguin S, Huang Y, Liu J, Wurtman R. Chronic administration of DHA and UMP improves the impaired memory of environmentally impoverished rats. Behav Brain Res 2008;191(1):11-6.

10. Olson AK, Eadie BD, Ernst C, Christie BR. Environmental enrichment and voluntary exercise massively increase neurogenesis in the adult hippocampus via dissociable pathways. Hippocampus 2006;16(3):250-60.

11. Elgadir MA, Salama M, Adam A. Carica Papaya as a source of natural medicine and its utilization in selected pharmaceutical applications. Int J Pharm Pharm Sci 2013;6(1):868-71.

12. Marais L, Stein DJ, Daniels WM. Exercise increases BDNF levels in the striatum and decreases depressive-like behavior in chronically stressed rats. Metab Brain Dis 2009;24(4):587-97.

13. Awizio AK, Onofri F, Benfenati F, Bonaccurso E. Influence of synapsin I on synaptic vesicles: An analysis by force-volume mode of the atomic force microscope and dynamic light scattering. Biophys $\mathrm{J}$ 2007;93(3):1051-60.

14. Micheva KD, Busse B, Weiler NC, O'Rourke N, Smith SJ. Singlesynapse analysis of a diverse synapse population: Proteomic imaging methods and markers. Neuron 2010;68(4):639-53.

15. Soontornniyomkij V, Risbrough VB, Young JW, Soontornniyomkij B, Jeste DV, Achim CL. Increased hippocampal accumulation of 
autophagosomes predicts short-term recognition memory impairment in aged mice. Age (Dordr) 2012;34(2):305-16.

16. Sharma S, Singh R, Kaur M, Kaur G. Late-onset dietary restriction compensates for age-related increase in oxidative stress and alterations of HSP 70 and synapsin 1 protein levels in male Wistar rats. Biogerontology 2010;11(2):197-209.

17. Li J, Zhou Y, Liu BB, Liu Q, Geng D, Weng LJ, et al. Nobiletin Ameliorates the Deficits in Hippocampal BDNF, TrkB, and Synapsin I Induced by Chronic Unpredictable Mild Stress. Evid Based Complement Alternat Med 2013;2013:359682.
18. Dunlop SA. Activity-dependent plasticity: Implications for recovery after spinal cord injury. Trends Neurosci 2008;31(8):410-8.

19. Devine JM, Zafonte RD. Physical exercise and cognitive recovery in acquired brain injury: A review of the literature. PM R 2009;1(6):560-75.

20. Zhou H, Zhang H, Cui J, Liu Y, Wu R, Xiang H. Protopanaxadiol saponins in the caudexes and leaves of panax notoginseng could be the main constituents that contribute to its antidepressant effects. Int J Pharm Pharm Sci 2014;6(11):301-11.

21. Kozlowski DA, Leasure JL, Schallert T. The control of movement following traumatic brain injury. Compr Physiol 2013;3(1):121-39. 